\section{COVID-19: unidades de terapia intensiva, ventiladores mecânicos e perfis latentes de mortalidade associados à letalidade no Brasil}

\author{
COVID-19: intensive care units, mechanical \\ ventilators, and latent mortality profiles \\ associated with case-fatality in Brazil
}

COVID-19: unidades de cuidados intensivos,
ventiladores mecánicos y perfiles latentes de
mortalidad asociados a la letalidad en Brasil
Rafael da Silveira Moreira 1,2

doi: 10.1590/0102-311X00080020

\section{Resumo}

O acelerado aumento do número de casos de doença pelo novo coronavírus (COVID-19) exige que os países aumentem as vagas nas unidades de terapia intensiva (UTI). Doenças respiratórias, neoplasias, cardiopatias, hipertensão e diabetes aumentam sua letalidade. O estudo objetivou identificar tanto as regiões com as maiores taxas de mortalidade específica por essas doenças quanto as com maior escassez de UTI e ventiladores pulmonares. Foi realizado um estudo ecológico transversal, as unidades de análise foram as Regiões de Saúde no Brasil. A fonte de dados foi o Departamento de Informática do SUS DATASUS (Cadastro Nacional de Estabelecimentos de Saúde - 2019, Sistemas de Informação de Mortalidade - 2017 e Projeções Populacionais - 2017). Foram calculadas as taxas por 100 mil habitantes de mortalidade específica para hipertensão, neoplasias, diabetes, doenças cardíacas e respiratórias, leitos de UTI total, leitos de UTI privados, leitos de UTI do Sistema Único de Saúde (SUS) e ventiladores do SUS. O perfil de mortalidade foi determinado pela análise de perfis latentes, e a análise de clusters dos leitos e ventiladores foi feita pelo método de varredura espacial. Mapas de Kernel foram construídos para a visualização dos dados. O nível de significância foi de 5\%. Observou-se quatro perfis latentes de mortalidade. As regiões de saúde com as maiores médias na mortalidade estão localizadas em regiões cuja escassez de leitos de UTI $e$ de ventiladores foi visualizada, especialmente, em partes das regiões Nordeste, Sudeste e Sul. A localização espacial das regiões com maior mortalidade e com escassez de leitos de UTI/ventiladores requer a atenção dos gestores e planejadores públicos, para o enfrentamento eficiente e equânime da epidemia no Brasil.

COVID-19; Unidades de Terapia Intensiva; Ventiladores Mecânicos; Análise de Classes Latentes; Mortalidade

\author{
Correspondência \\ R. S. Moreira \\ Instituto Aggeu Magalhães, Fundação Oswaldo Cruz. \\ Av. Professor Moraes Rego s $/ n$, Cidade Universitária, Recife, PE \\ 50670-420, Brasil. \\ moreirars@cpqam.fiocruz.br \\ 1 Instituto Aggeu Magalhães, Fundação Oswaldo Cruz, Recife, \\ Brasil. \\ 2 Faculdade de Medicina, Universidade Federal de Pernambuco, \\ Recife, Brasil.
}




\section{Introdução}

O surto da infecção pelo coronavírus da síndrome respiratória aguda grave 2 (SARS-CoV-2), nomeada como COVID-19 (em inglês coronavirus disease 2019), foi inicialmente relatado em dezembro de 2019, em Wuhan, China 1,2. Essa doença surgiu associada a formas graves de pneumonia, com rápida disseminação humana. Os pacientes apresentam tosse seca, dor de cabeça, hipóxia, febre e dispneia. As mortes ocorrem devido a uma falência respiratória progressiva causada por danos pulmonares 3,4,5. Dessa forma, casos graves geram cuidados em unidades de terapia intensiva (UTI) 6 .

A elevação rápida dos números de casos e óbitos na China levou a Organização Mundial da Saúde (OMS) a decretar em 30 de janeiro de 2020 uma Emergência em Saúde Pública de Interesse Internacional 7. A partir desse momento, observou-se uma sequência de dispositivos sanitários sendo requisitados pela OMS até chegarmos ao status de pandemia mundial decretada em 11 de março de 20208.

A distribuição espacial da doença ultrapassou várias fronteiras nacionais, levando diversos países a decretarem emergências e estados de calamidade pública. Com destaque para a mudança do epicentro da doença da China para a Europa, em especial Itália e Espanha. Em março de 2020, os Estados Unidos também passaram a contribuir com um número cada vez maior de casos e óbitos pela COVID-19.

No Brasil, o primeiro caso foi identificado em 26 de fevereiro de 2020 no Estado de São Paulo, e o primeiro óbito em 17 de março de 2020, no mesmo estado. As estimativas de progressão da epidemia são de crescimento exponencial (Ministério da Saúde. Painel coronavirus. https://covid.saude.gov. br/, acessado em 12/Abr/2019). Medidas de contenção do avanço incluem cuidados com higiene, a testagem do maior número de casos e o isolamento social ${ }^{9}$, haja vista ser uma doença que pode não expressar sintomas em seus portadores 10,11,12.

Todas as medidas tomadas até o momento visam a evitar a superação da capacidade dos sistemas de saúde em atender à população que evolui para as formas mais graves da doença. Nessas situações, são necessárias internações em UTI e o uso de ventiladores pulmonares para o suporte respiratório desses casos 13. Nesse sentido, o que observamos desde o início da epidemia na China, foram os diversos países se mobilizando no intuito de prover seus sistemas de saúde com a máxima capacidade de atendimento dos pacientes que apresentarem complicações respiratórias. Entrementes, os casos mais graves têm sido registrados em pacientes mais velhos e que apresentam algum tipo de comorbidade, especialmente doenças respiratórias, cardíacas, hipertensão e diabetes 14. Contudo, essa associação com outras comorbidades torna a população mais jovem portadora dessas condições também um grupo de risco.

Como não possuímos um cadastro das pessoas que apresentam essas comorbidades sem incorrer em uma considerável subnotificação, o uso das informações de mortalidade por essas causas pode indicar um cenário em que elas estão presentes em maior número e com maior fidedignidade. Nesse sentido, o objetivo deste estudo epidemiológico foi identificar as regiões de saúde no Brasil com as maiores taxas de mortalidade por doenças associadas às formas graves da COVID-19, considerando-se a distribuição espacial da cobertura de suporte hospitalar (leitos de UTI e ventiladores pulmonares) para o enfrentamento nacional da pandemia.

\section{Metodologia}

Foi realizado um estudo ecológico espacial transversal. As unidades de análise foram as Regiões de Saúde no Brasil. Segundo o Ministério da Saúde, conceitua-se Região de Saúde como o espaço geográfico contínuo, formado pela união de municípios limítrofes, segundo identidades culturais, econômicas e sociais. Além disso, consideram-se as redes de comunicação e infraestrutura de transportes compartilhados, com o objetivo de integrar a organização, o planejamento e a execução de ações e serviços de saúde 15 . Nesse sentido, fizeram parte deste trabalho todas as 450 Regiões de Saúde do Brasil.

Foram utilizados dois blocos de variáveis: o bloco das variáveis de mortalidade e o bloco das variáveis de cobertura assistencial. No bloco das variáveis de mortalidade, foram selecionadas as principais causas de mortalidade relacionadas à forma grave da COVID-19. Nesse sentido, foram calculados quatro conjuntos de taxas de mortalidade específicas por 100 mil habitantes no ano de 2017, por ser o período mais recente em que tínhamos disponibilizados os dados pelo site do Departamento de 
Informática do SUS (DATASUS. http://www.datasus.gov.br). Foram usados os óbitos por residência e a estimativa populacional para o mesmo ano, segundo o Tribunal de Contas da União (TCU). Dessa forma, foram calculadas as taxas de mortalidade do grupo de doenças neoplásicas (Capítulo 2, CID-10), do aparelho respiratório (Capítulo 10, CID-10), diabetes (Capítulo 4, E10-E14, CID-10), hipertensão e doenças cardíacas (Capítulo 9, I10-I15, I20-I52, CID -10).

Para o bloco das variáveis da cobertura assistencial foram usadas quatro taxas de cobertura populacional (por 100 mil habitantes): o número de UTI adultos total dividido pela população total, o número de UTI adultos do Sistema Único de Saúde (SUS) dividido pela população SUS-dependente (população não coberta por plano de saúde), o número de UTI adultos no setor privado dividido pela população não SUS-dependente e o número de ventiladores pulmonares do SUS dividido pela população SUS-dependente. Os dados são de janeiro de 2020, disponíveis pelo Cadastro Nacional de Estabelecimentos de Saúde (CNES). Todos os dados referentes a esse bloco de variáveis foram publicamente disponibilizados pelo Instituto de Estudos para Políticas de Saúde, em sua Nota Técnica no 3, de 202013

A análise dos dados foi realizada em três blocos. No primeiro, foi construído o perfil de mortalidade segundo os quatro conjuntos de causas. Nesse sentido, foi realizada a análise de perfis latentes (APL) 16,17,18, uma metodologia de análise estatística que busca agrupar o conjunto de dados (qualitativos ou quantitativos) segundo semelhanças no perfil de resposta. Busca-se uma homogeneidade intragrupo e uma heterogeneidade intergrupo. A variável latente é uma variável que não se observa diretamente, mas indiretamente, com base naquelas que podem ser observadas diretamente (variáveis indicadoras). Como não existe uma única condição de morbidade que predispõe os indivíduos à forma grave da COVID-19, com base nos principais grupos de mortalidade, foi construída uma única variável latente que englobasse simultaneamente esses quatro grupos.

O objetivo da APL é classificar indivíduos de uma população heterogênea para subgrupos menores e mais homogêneos, com base nos valores dos indivíduos em variáveis contínuas. É importante observar que a APL não se limita às variáveis contínuas, mas pode incluir combinações de contínua, discretas e categóricas como variáveis indicadoras das classes latentes 19 . Embora essa opção seja distinta da análise de classes latentes (ACL), que considera apenas as variáveis indicadoras categóricas, ambas as análises geram como resultado classes latentes (no caso de ACL) e perfis latentes (no caso de APL) categóricas 20.

Na construção dessa variável latente, modelos com diferentes números de perfis latentes (categorias) foram criados e testados até se encontrar o modelo ideal para descrever essa variável. Na escolha do melhor modelo estatístico da variável latente foram observados os seguintes critérios: critério de informação de Akaike (AIC), critério de informação Bayesiano (BIC) e critério de informação Bayesiano ajustado (BIC ajustado), observando-se sempre os menores valores quando comparados o modelo atual com o anterior. Também foi considerado o maior valor da entropia 21 . Além desses critérios, foram realizados outros três testes estatísticos (Vuong-Lo-Mendell-Rubin; Lo-Mendell-Rubin; Parametric bootstrapped) para verificar se o número de perfis escolhido é melhor em termos de ajuste do modelo, quando comparado com o número de perfis do modelo anterior. Para esta análise foi utilizado o programa Mplus 6.12 (https://www.statmodel.com/version6.12.shtml).

Para a análise da cobertura assistencial foi realizado o teste de varredura espacial na identificação de clusters (agrupamentos) espaciais. Dessa forma, as quatro taxas de coberturas foram recalculadas para a construção de taxas esperadas considerando a população exposta e sua matriz de vizinhança, segundo determinado raio de varredura espacial (janela espacial). Uma vez tendo em mãos as taxas esperadas, foram calculadas as razões de cobertura entre a taxa observada (real) e a taxa esperada em cada Região de Saúde. Valores acima de 1 indicam clusters onde existe uma taxa de cobertura acima do esperado, ao passo que valores abaixo de 1 indicam clusters onde as taxas observadas estão abaixo do que seria esperado.

Nessa análise, foi utilizado o programa SaTScan 9.6 (https://www.satscan.org/), com um modelo de probabilidade de Poisson 22, para a detecção de clusters de alto e baixo riscos e janela espacial circular com $15 \%$ da população exposta sob risco. Essa porcentagem levou em consideração o fato de que não são todos os indivíduos infectados pela COVID-19 que precisarão de internação em unidades de UTI, mas cerca de $15 \%$ que apresentam a forma severa da doença 14 . Foi usado o teste de máxima verossimilhança para identificar aglomerados. A hipótese alternativa é a de que existe um valor elevado no 
interior da janela em comparação com o exterior. Foram consideradas simulações de Monte Carlo (999 permutações) para a obtenção dos valores de p ${ }^{22}$. O nível de significância adotado foi de 5\%.

Após a análise dos dois blocos foi realizada a construção de mapas de Kernel para a visualização espacial das regiões de saúde segundo o perfil de mortalidade e o perfil de cobertura assistencial. Os mapas de Kernel ou estimadores de Kernel são um conjunto de procedimentos estatísticos não paramétricos de suavização de pontos em uma superfície geográfica de acordo com a densidade destes pontos 23. Trata-se do ajuste de uma função bidimensional que realiza uma contagem de todos os pontos dentro de uma área de influência (grade), ponderando-os pela distância de cada um à localização de interesse. O grau de alisamento utilizou um raio adaptativo, variando de acordo com a densidade dos pontos e função quártica. Os pontos foram gerados baseando-se nos centroides das Regiões de Saúde. A análise espacial foi realizada com o auxílio do programa TerraView 4.2.2 (http://www.dpi. inpe.br/terraview). Essa técnica estatística produz uma superfície de densidade em que é possível observar a concentração de fenômenos que indicam uma aglomeração espacial 24. Em todos os blocos de análise o nível de significância adotado nas inferências estatísticas foi de $5 \%$.

Por serem dados publicamente disponibilizados na Internet e sem nenhuma forma e identificação individual, sendo agregados por Regiões de Saúde, este estudo não precisou ser submetido ao Comitê de Ética em Pesquisas com seres humanos.

\section{Resultados}

A Tabela 1 mostra os resultados da APL. Segundo os parâmetros analisados, o melhor modelo foi o que apresenta quatro perfis latentes de mortalidade. Embora não tenha apresentado o maior valor de entropia, foi o modelo que apresentou os menores valores de AIC, BIC e BIC ajustado, associado com a significância estatística dos três testes analisados. Esses três testes indicam que o modelo com quatro perfis é superior e ao modelo antecessor de três perfis. Também podemos observar na Tabela 1 a tipologia do perfil de mortalidade, gerada pela APL, segundo as médias das taxas de mortalidade em cada perfil por causa específica. Observamos uma variável qualitativa ordinal em que no perfil 1 encontramos as menores taxas e no perfil 4 as maiores.

$\mathrm{Na}$ Tabela 1, também são apresentadas as probabilidades diagonais para cada perfil. Essa informação refere-se à probabilidade de cada indivíduo classificado no seu perfil pertencer de fato a este perfil. Uma classificação perfeita seria de $100 \%$ de probabilidade. Contudo, a grande contribuição dessa análise é chegar o mais próximo possível de um padrão que potencializa o maior valor possível de concordância classificatória. Trata-se de uma alternativa aos tradicionais pontos de corte que sempre penalizam os valores limítrofes. Nesse sentido, a probabilidade diagonal traz essa informação na qual as classificações não são perfeitas, mas indicam qual a probabilidade de acerto em sua discriminação intergrupal. Assim, cada perfil significa um bloco de Regiões de Saúde com semelhanças nas taxas de mortalidade específicas dos quatro grandes grupos de mortalidade (neoplasia, respiratórias, diabetes, hipertensão/doenças cardíacas). O perfil é analisado pela média de cada coeficiente de mortalidade presente em cada um dos quatro perfis latentes. Dessa forma, o perfil de mortalidade ficou definido como uma variável qualitativa ordinal. O perfil 1 foi o conjunto de Regiões de Saúde com coeficientes baixos, o perfil 2 com valores médios, o perfil 3 com coeficientes altos e o perfil 4 com coeficientes muito altos.

Com relação ao número total de UTI, o Brasil contava com 29.891 unidades, sendo 14.094 UTI do SUS e 15.797 de UTI privada. O SUS tinha 40.508 ventiladores mecânicos. Das 450 Regiões de Saúde, 126 não tinham UTI, seja do SUS ou privada, com 44,4\% na Região Nordeste. Outras 145 Regiões de Saúde não contavam com UTI do SUS, também com predominância na Região Nordeste (45,5\%). Encontrou-se 188 Regiões de Saúde sem UTI privadas, sendo 42\% na Região Nordeste.

Outro dado que chama a atenção na Tabela 1 são as Regiões de Saúde classificadas no pior perfil de mortalidade (perfil 4), em que 42,9\% e 36,7\% delas têm, respectivamente, taxas de leitos de UTI total e de UTI privada aquém do esperado.

A Tabela 2 apresenta os resultados dos clusters, com significância estatística, referentes às taxas assistenciais (UTI e ventiladores). Destaca-se a Região Nordeste, onde cerca de 70\% de suas Regiões de Saúde têm uma taxa de UTI total aquém do esperado. Resultado semelhante com relação às UTI do 
Tabela 1

Classificação dos perfis de mortalidade segundo a análise de perfis latentes e segundo os clusters espaciais de valores aquém e além das taxas assistenciais (UTI total, UTI SUS, UTI privada e ventiladores respiratórios do SUS). Brasil, 2020.

\begin{tabular}{|c|c|c|c|c|c|}
\hline \multirow[t]{2}{*}{ Perfil de mortalidade } & \multicolumn{4}{|c|}{ Análise de perfis latentes } & \multirow[t]{2}{*}{ Total } \\
\hline & Perfil 1 & Perfil 2 & Perfil 3 & Perfil 4 & \\
\hline Classificação da mortalidade & Baixo & Médio & Alto & Muito alto & \\
\hline \multirow[t]{2}{*}{ Número de observações (Regiões de Saúde) } & 90 & 86 & 176 & 98 & 450 \\
\hline & Média (DP) & Média (DP) & Média (DP) & Média (DP) & Média (DP) \\
\hline \multicolumn{6}{|l|}{ Coeficiente médio por 100 mil habitantes } \\
\hline Neoplasia & $61,2(17,9)$ & $77,0(14,2)$ & $113,1(20,1)$ & $138,5(28,4)$ & $101,6(35,3)$ \\
\hline Respiratórias & $44,4(13,8)$ & $55,4(12,2)$ & $87,3(16,4)$ & $103,6(17,0)$ & $76,3(27,3)$ \\
\hline Diabetes & $22,5(6,7)$ & $43,3(9,0)$ & $28,0(7,0)$ & $41,1(9,6)$ & $32,9(11,8)$ \\
\hline Hipertensão e cardiopatias & $71,3(17,3)$ & $116,9(18,7)$ & $116,3(17,5)$ & $151,6(21,1)$ & $115,6(32,4)$ \\
\hline \multirow[t]{2}{*}{ Probabilidades diagonais } & 0,92 & 0,89 & 0,88 & 0,90 & - \\
\hline & n (\%) & n (\%) & n (\%) & n (\%) & n (\%) \\
\hline \multicolumn{6}{|l|}{ Clusters assistenciais } \\
\hline Clusters UTI total aquém do esperado & $37(41,1)$ & $55(64,0)$ & $79(44,9)$ & $42(42,8)$ & $213(47,3)$ \\
\hline Clusters UTI total além do esperado & $2(2,2)$ & $1(1,1)$ & $11(6,2)$ & $9(9,2)$ & $23(5,1)$ \\
\hline Sem significância estatística UTI total & $51(56,7)$ & $30(34,9)$ & $86(48,9)$ & $47(48,0)$ & $214(47,6)$ \\
\hline Clusters UTI SUS aquém do esperado & $21(23,3)$ & $48(55,8)$ & $13(7,4)$ & $10(10,2)$ & $92(20,4)$ \\
\hline Clusters UTI SUS além do esperado & $1(1,1)$ & $4(4,7)$ & $26(14,8)$ & $9(9,2)$ & $40(8,9)$ \\
\hline Sem significância estatística UTI SUS & $68(75,6)$ & $34(39,5)$ & $137(77,8)$ & $79(80,6)$ & $318(70,7)$ \\
\hline Clusters UTI privado aquém do esperado & $12(13,3)$ & $13(15,1)$ & $66(37,5)$ & $36(36,7)$ & $127(28,2)$ \\
\hline Clusters UTI privado além do esperado & $10(11,1)$ & $16(18,6)$ & $12(6,8)$ & $8(8,2)$ & $46(10,2)$ \\
\hline Sem significância estatística UTI privado & $68(75,6)$ & $57(66,3)$ & $98(55,7)$ & $54(55,1)$ & $277(61,6)$ \\
\hline Clusters ventiladores SUS aquém do esperado & $71(78,9)$ & $67(77,9)$ & $40(22,7)$ & $17(17,3)$ & $195(43,3)$ \\
\hline Clusters ventiladores SUS além do esperado & $3(3,3)$ & $1(1,2)$ & $39(22,2)$ & $12(12,2)$ & $55(12,2)$ \\
\hline Sem significância estatística ventiladores & $16(17,8)$ & $18(20,9)$ & $97(55,1)$ & $69(70,4)$ & $200(44,5)$ \\
\hline
\end{tabular}

DP: desvio padrão; SUS: Sistema Único de Saúde; UTI: unidade de terapia intensiva.

SUS, em que cerca de metade das Regiões de Saúde dessa região (55,6\%) contam com leitos aquém do esperado. Entretanto, quando se consideram leitos de UTI privados, a Região Nordeste registra 27,1\% de suas Regiões de Saúde com taxas além do esperado. No que se refere aos ventiladores do SUS, embora praticamente todas as Regiões de Saúde tenham as taxas aquém do esperado, essas taxas são de 93,3\% na Região Norte e 81,2\% na Região Nordeste. Ressalta-se que os valores sem significância estatística (Tabelas 1 e 2) correspondem às Regiões de Saúde onde a razão entre as taxas observadas e as esperadas não foram significantes, segundo os parâmetros de varredura executados pela janela espacial utilizada.

A Figura 1 apresenta os mapas de Kernel das Regiões de Saúde segundo a categoria do perfil de mortalidade estimada pela APL. Observamos que o perfil 1 está concentrado nas regiões Norte e Nordeste, com destaque para o norte dos estados do Maranhão, Bahia e Pará, e noroeste do Mato Grosso. Na Região Sudeste foi encontrada uma alta densidade de regiões em grande parte do Estado de Minas Gerais. O padrão do perfil 2 foi fortemente concentrado na região leste dos estados da Paraíba, Pernambuco e Alagoas, alcançando grande parte dos outros estados do Nordeste. Também observou-se maior densidade em Tocantins e sudoeste do Mato Grosso. Os perfis 3 e 4 apresentaram um padrão semelhante, com fortes densidades em vários estados do Nordeste, Sudeste e Sul. O pior perfil (perfil 4) foi na região noroeste do Estado de São Paulo e centro-norte do Rio Grande do Sul. 


\section{Tabela 2}

Distribuição dos clusters espaciais de valores das taxas assistenciais (UTI total, UTI SUS, UTI privada e ventiladores respiratórios do SUS) aquém e além, segundo as regiões geográficas. Brasil, 2020.

\begin{tabular}{|c|c|c|c|c|c|c|}
\hline & $\begin{array}{l}\text { Norte } \\
\text { n (\%) }\end{array}$ & $\begin{array}{c}\text { Nordeste } \\
\text { n (\%) }\end{array}$ & $\begin{array}{c}\text { Sudeste } \\
\text { n (\%) }\end{array}$ & $\begin{array}{c}\text { Sul } \\
\text { n (\%) }\end{array}$ & $\begin{array}{c}\text { Centro-oeste } \\
\text { n (\%) }\end{array}$ & $\begin{array}{l}\text { Total } \\
\text { n (\%) }\end{array}$ \\
\hline Número de Regiões de Saúde & 45 & 133 & 165 & 68 & 39 & 450 \\
\hline Clusters UTI total aquém do esperado & $14(31,1)$ & $94(70,7)$ & $59(35,8)$ & $43(63,2)$ & $3(7,7)$ & $213(47,3)$ \\
\hline Clusters UTI total além do esperado & $0(0,0)$ & $0(0,0)$ & $13(7,9)$ & $6(8,8)$ & $4(10,2)$ & $23(5,1)$ \\
\hline Sem significância estatística UTI total & $31(68,9)$ & $39(29,3)$ & $93(56,4)$ & $19(28,0)$ & $32(82,1)$ & $214(47,6)$ \\
\hline Clusters UTI SUS aquém do esperado & $9(20,0)$ & $74(55,6)$ & $4(2,4)$ & $0(0,0)$ & $5(12,8)$ & $92(20,4)$ \\
\hline Clusters UTI SUS além do esperado & $0(0,0)$ & $1(0,8)$ & $34(20,6)$ & $3(4,4)$ & $2(5,1)$ & $40(8,9)$ \\
\hline Sem significância estatística UTI SUS & $36(80,0)$ & $58(43,6)$ & $127(77,0)$ & $65(95,6)$ & $32(82,1)$ & $318(70,7)$ \\
\hline Clusters UTI privado aquém do esperado & $0(0,0)$ & $18(13,5)$ & $66(40,0)$ & $43(63,2)$ & $0(0,0)$ & $127(28,2)$ \\
\hline Clusters UTI privado além do esperado & $2(4,4)$ & $36(27,1)$ & $7(4,2)$ & $0(0,0)$ & $1(2,6)$ & $46(10,2)$ \\
\hline Sem significância estatística UTI privado & $43(95,6)$ & $79(59,4)$ & $92(55,8)$ & $25(36,8)$ & $38(97,4)$ & $277(61,6)$ \\
\hline Clusters ventiladores SUS aquém do esperado & $42(93,3)$ & $108(81,2)$ & $28(17,0)$ & $0(0,0)$ & $17(43,6)$ & $195(43,3)$ \\
\hline Clusters ventiladores SUS além do esperado & $0(0,0)$ & $1(0,8)$ & $50(30,3)$ & $2(2,9)$ & $2(5,1)$ & $55(12,2)$ \\
\hline Sem significância estatística ventiladores & $3(6,7)$ & $24(18,0)$ & $87(52,7)$ & $66(97,1)$ & $20(51,3)$ & $200(44,5)$ \\
\hline
\end{tabular}

SUS: Sistema Único de Saúde; UTI: unidade de terapia intensiva.

A Figura 2 apresenta os mapas de Kernel em que estão localizadas as regiões onde as taxas de cobertura estão aquém do que seria o esperado. Observamos que as regiões Norte e Centro-oeste são as que apresentam grande número de Regiões de Saúde onde não foram encontrados clusters significantes. Considerando-se as regiões que apresentam clusters significantes de leitos de UTI e de ventiladores pulmonares do SUS, observa-se um cenário no qual as principais Regiões de Saúde que registram escassez na oferta dos cuidados intensivos, também se sobrepõe à severidade do perfil de mortalidade por doenças associadas à maior letalidade por COVID-19. Considerando-se a escassez de UTI total e exclusiva pelo SUS, grande parte das regiões Nordeste, Sudeste e Sul sofre desta escassez, ao mesmo tempo em que concentram várias regiões classificadas como perfis 3 e 4 (piores cenários de mortalidade, Figura 1).

Chama a atenção o fato de que grande parte da Região Norte foi classificada como perfil 1 de mortalidade (Figura 1). Essa Região também não apresentou clusters significantes de escassez de leitos de UTI, exceto com relação aos clusters de ventiladores do SUS. Contudo, vale destacar o tamanho da população exposta pela janela espacial de varredura nessa Região. Devido à pouca densidade demográfica, os valores baixos observados estavam dentro do esperado ao se considerar um raio que necessita de maior diâmetro para alcançar 15\% da população exposta.

\section{Discussão}

O uso de perfis latentes revelou regiões no Brasil onde as principais causas de mortalidade associadas à maior letalidade por COVID-19 estão presentes. Um estudo realizado no primeiro epicentro da epidemia, em Hubei na China 25 , mostrou que a média de idade dos pacientes admitidos na UTI era de 66 anos, com predominância masculina, e cerca de 58\% apesentavam condições crônicas, incluindo hipertensão, diabetes, problemas cardíacos e insuficiência renal. Segundo o estudo, esses indivíduos apresentam maior probabilidade de evoluir para as formas graves da doença. De acordo com Wu \& McGoogan 14, a taxa de letalidade é elevada em 10,5\% para doenças cardiovasculares, 7,3\% para diabéticos, 6,3\% para doenças respiratórias crônicas e 6\% para pacientes hipertensos. 
Figura 1

Mapas de Kernel das Regiões de Saúde segundo os perfis latentes de mortalidade por doenças associadas à maior letalidade pela COVID-19. Brasil, 2020.

1a) Perfil 1: baixo

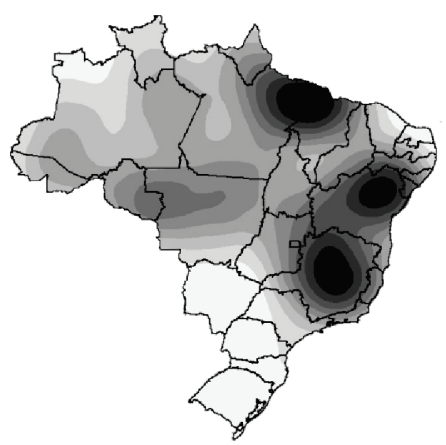

1c) Perfil 3: alto

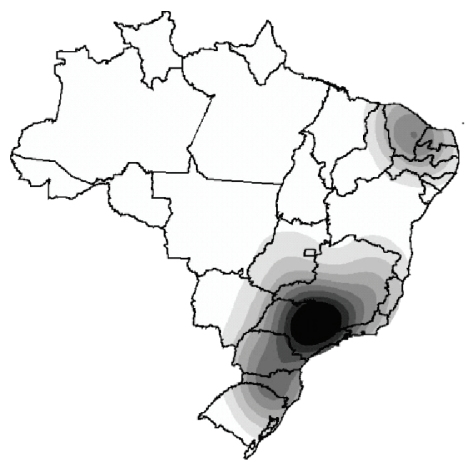

1b) Perfil 2: médio

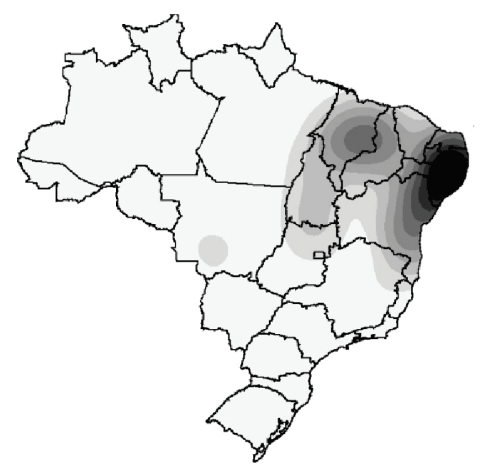

1d) Perfil 4: muito alto

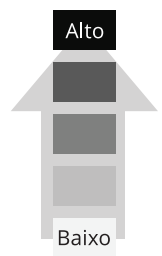

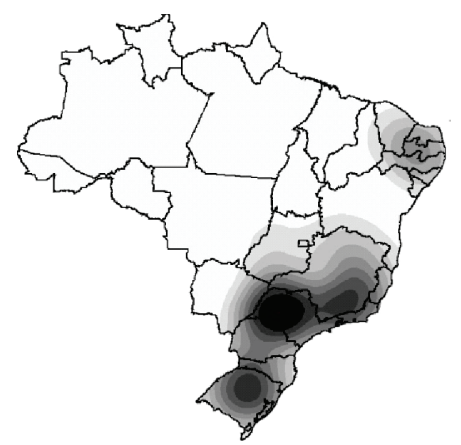

SUS: Sistema Único de Saúde; UTI: unidade de terapia intensiva.

Dessa maneira, consideramos ser de grande auxílio a identificação espacial das regiões onde a presença simultânea desses quatro conjuntos de causas específicas de mortalidade ocorre. A APL permitiu localizar regiões onde as taxas de mortalidade são semelhantes. Por seu turno, o mapa de Kernel possibilitou a visualização espacial onde esses perfis ocorrem. Nesse sentido, o espaço se configura como um dos braços da epidemiologia na medida em que permite o conhecimento não só do lugar onde os eventos em saúde ocorrem, mas também estabelecer o papel que o espaço tem na geração destes eventos. Conhecer quem sofre e onde sofrem constituem as variáveis principais, tanto no estabelecimento de relações causais quanto no estabelecimento de ações de saúde pública.

No que tange à distribuição espacial das taxas de coberturas assistenciais, vale destacar antes algumas questões relevantes para o enfrentamento da pandemia. Inicialmente, reforçar que as medidas de prevenção adotadas parecem ser ainda, mesmo depois da humanidade ter experimentado situações semelhantes às de pandemia, as principais armas de combate. Chama a atenção, mesmo após avanços tecnológicos e científicos, ainda sermos levados a adotar as medidas básicas de higiene, quarentena, isolamento e distanciamento social historicamente recomendadas em epidemias do passado 26. Adicionalmente, serão também essas medidas que determinarão o número exato de pessoas com sintomas severos da doença 27 que, por sua vez, determinará a demanda por ventiladores pulmonares e leitos de UTI. 
Figura 2

Mapas de Kernel dos clusters das taxas por 100 mil habitantes de UTI total, UTI SUS, UTI privado e ventiladores do SUS aquém do esperado. Brasil, 2020.

2a) UTI total

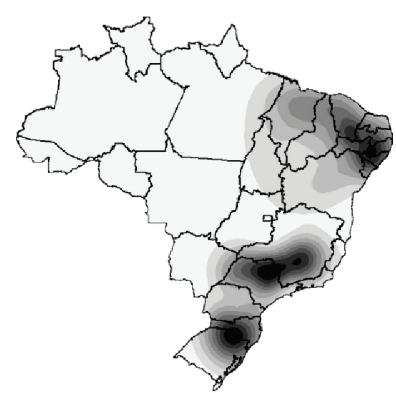

2c) UTI privado

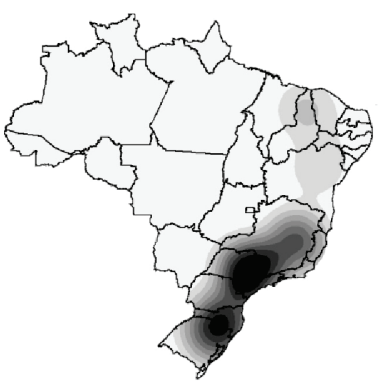

2b) UTI SUS

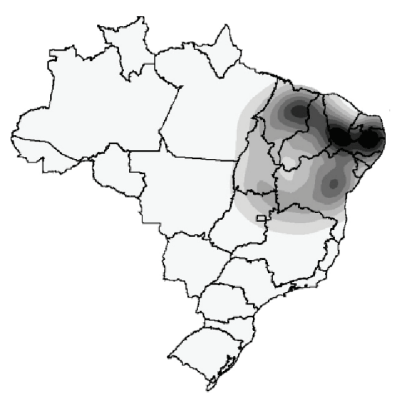

2d) Ventiladores do SUS

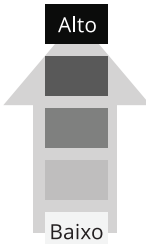

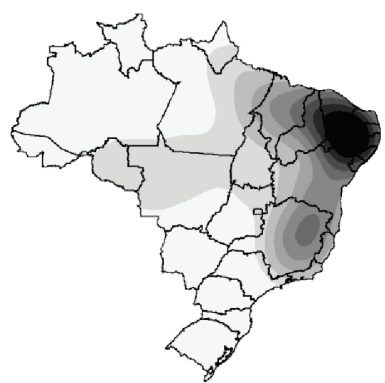

SUS: Sistema Único de Saúde; UTI: unidade de terapia intensiva.

Diante da escassez de recursos médicos assistenciais, incluindo tanto recursos físicos quanto humanos, surge a questão sobre o seu uso e alocamento de forma justa. Propostas anteriores para a alocação de recursos em pandemias e outros ambientes de escassez absoluta convergem em quatro valores fundamentais: maximizar os benefícios produzidos por recursos escassos, tratar as pessoas igualmente, promover e recompensar o valor instrumental, e dar prioridade para o pior momento. Existe um consenso de que a classe social de uma pessoa não deve determinar quem vive ou morre. Embora o tratamento médico nos Estados Unidos fora de contextos de pandemia seja frequentemente restrito àqueles que podem pagar, nenhuma proposta apoia a capacidade de pagamento como critério em uma pandemia 27.

As intervenções críticas tais como os testes, equipamentos de proteção individual (EPI), leitos de UTI, ventiladores, terapêutica e vacinas, devem ir primeiro aos profissionais de saúde da linha de frente e a outros que cuidam de pacientes doentes e que mantêm a infraestrutura crítica em operação. Em particular os trabalhadores que enfrentam um alto risco de infecção e cujo treinamento dificulta sua substituição, devem ser priorizados. Esses trabalhadores devem ter prioridade não porque sejam de alguma forma mais dignos, mas por causa de seu valor instrumental: eles são essenciais para a resposta à pandemia 28,29 . No entanto, o fato de estarem na linha de frente aumenta consideravelmente o risco de infecção, especialmente na ausência de EPI e de protocolo adequado de trabalho 30 . A neces- 
sidade de equilibrar múltiplos valores éticos para várias intervenções e em diferentes circunstâncias provavelmente levará a julgamentos diferentes sobre quanto peso dar a cada valor em casos particulares. Isso destaca a necessidade de procedimentos de alocação justos e consistentes que incluam as partes afetadas. Esses procedimentos devem ser transparentes para garantir a confiança do público em sua justiça 27.

Com relação às prioridades para a ocupação dos leitos de UTI no Brasil, o Conselho Federal de Medicina (CFM) elaborou a Resolução no 2.156/2016. Nessa Resolução, o critério para a admissão em UTI é a instabilidade crítica, e passa pela necessidade de intervenção de suporte à vida, ausência de limitação de suporte e alta probabilidade de recuperação. A Resolução estabelece cinco níveis de prioridade que vão desde a prioridade máxima que considera pacientes que necessitam de intervenções de suporte à vida, com alta probabilidade de recuperação e sem nenhuma limitação de suporte terapêutico, até a última prioridade relativa aos pacientes com doença em fase de terminalidade, ou moribundos, sem possibilidade de recuperação 31 .

Contudo, esses critérios não foram construídos pensando em uma situação de pandemia como a que estamos vivendo. Podemos ter situações com vários pacientes na categoria de prioridade máxima. Critérios sociais podem levar a diversas leituras distintas, gerando preconceitos, com exceção da classe dos profissionais de saúde pelos motivos já citados. Wang \& de Lucca-Silveira 31 consideram algumas alternativas. Uma delas seria alterar os critérios de desinternação, dando alta para pacientes de forma prematura, de modo a permitir maior poder de rotatividade dos leitos. Além disso, é necessário considerar que podem existir outras condições que demandam o uso de UTI, nas situações rotineiras, que não são causadas pela COVID-19. Com efeito, os sistemas de atenção primária e secundária devem atuar de forma efetiva para evitar o encaminhamento ao cuidado intensivo. Por fim, embora cada hospital estabeleça seus critérios próprios para a distribuição de vagas na UTI, deve-se considerar que em momentos de tragédia, de escassez de recursos, a centralização da decisão no uso dos leitos de UTI pode ser uma alternativa logisticamente válida. Considerando-se a existência de leitos privados, o que poderia gerar uma determinação social na disponibilidade destes leitos, a administração em âmbito federal dos mesmos, seja de forma colaborativa ou compulsória, pode ajudar na homogeneização dos critérios e na equidade distributiva dos leitos.

Com efeito, o fato de a Região Norte e parte do Centro-oeste não apresentarem clusters significantes de taxas assistenciais abaixo do esperado não significa a presença de melhor arsenal assistencial. A grande dimensão geográfica dessas duas regiões, combinada com a baixa densidade demográfica, pode explicar a não formação de clusters, como mostrado nos Resultados. Assim, regiões onde não existem leitos de UTI e ventiladores podem estar dentro do esperado segundo o padrão de varredura espacial. Da mesma forma, essas regiões apresentaram melhores perfis de mortalidade, o que mostra um cenário epidemiológico distinto com relação às doenças crônicas. Uma análise focada nos grandes centros urbanos dessas duas regiões geográficas poderia melhor avaliar a escassez de recursos assistenciais, independentemente de seu perfil epidemiológico de mortalidade. $\mathrm{O}$ fator mais importante na oferta assistencial para o enfrentamento da COVID-19 é o entendimento da sua acelerada taxa de infecção, na ausência de medidas de isolamento social.

Embora não seja uma limitação do estudo, o desenho ecológico não permite que inferências no nível individual sejam estabelecidas. Outro fator importante é a transversalidade do estudo não permitir a realização de hipóteses causais, contudo, o objetivo não foi estabelecer relações causais, mas de associação e prognóstico assistivo. O conhecimento espacial das ocorrências de mortalidade e de cobertura assistencial intensiva pode revelar locais onde intervenções são necessárias, de modo a evitar que a chegada massiva da COVID-19 no Brasil traga um colapso no seu SUS. 


\section{Conclusão}

Na pandemia da COVID-19 o conhecimento do arsenal médico-assistivo no Brasil é fundamental para o uso racional dos leitos de UTI e ventiladores pulmonares. Associado às regiões com maior probabilidade de demanda, determinada pelo perfil de mortalidade por causas que aumentam a letalidade do vírus, o que observamos é uma situação preocupante no tocante à resposta dos serviços de saúde. Mesmo com todos os cuidados na prevenção da disseminação, este estudo revela onde o poder sanitário público deve atuar no aumento da cobertura assistencial, com estratégias de racionamento desses equipamentos assistivos e critérios transparentes e justos na sua alocação. Nesse sentido, a análise espacial das duas dimensões estudadas pode ser útil na tomada de decisões dos gestores em saúde pública, em uma ação coordenada pela economia de escala e equidade distributiva.

\section{Informação adicional}

ORCID: Rafael da Silveira Moreira (0000-00030079-2901).

\section{Agradecimentos}

Ao Instituto de Estudos para Políticas de Saúde (IEPS) pela disponibilização pública de parte dos dados sistematizados usados neste estudo.

\section{Referências}

1. Zhu N, Zhang D, Wang W, Li X, Yang B, Song $\mathrm{J}$, et al. A novel coronavirus from patients with pneumonia in China, 2019. N Engl J Med 2020; 382:727-33.

2. World Health Organization. Novel coronavirus: China. http://www.who.int/csr/don/12january-2020-novel-coronavirus-china/en/ (acessado em 12/Abr/2020).

3. Tsang KW, Ho PL, Ooi GC, Yee WK, Wang $\mathrm{T}$, Chan-Yeung M, et al. A cluster of cases of severe acute respiratory syndrome in Hong Kong. N Engl J Med 2003; 348:1975-83.

4. Drosten C, Günther S, Preiser W, van der Werf S, Brodt HR, Becker S, et al. Identification of a novel coronavirus in patients with severe acute respiratory syndrome. N Engl J Med 2003; 348:1967-76.

5. Yang X, Yu Y, Xu J, Shu H, Xia J, Liu H, et al. Clinical course and outcomes of critically ill patients with SARS-CoV-2 pneumonia in $\mathrm{Wu}-$ han, China: a single-centered, retrospective, observational study. Lancet Respir Med 2020; [Epub ahead of print].

6. Chen N, Zhou M, Dong X, Qu J, Gong F, Han $\mathrm{Y}$, et al. Epidemiological and clinical characteristics of 99 cases of 2019 novel coronavirus pneumonia in Wuhan, China: a descriptive study. Lancet 2020; 395:507-13.

7. World Health Organization. Novel coronavirus (2019-nCoV). Situation report 11. https:// www.who.int/docs/default-source/corona viruse/situation-reports/20200131-sitrep11-ncov.pdf?sfvrsn=de7c0f7_4 (acessado em 12/Abr/2020). 
8. World Health Organization. Novel coronavirus (2019-nCoV). Situation report 51. https:// www.who.int/docs/default-source/corona viruse/situation-reports/20200311-sitrep51 - covid-19.pdf? sfvrsn=1ba62e57_10 (acessado em 12/Abr/2020).

9. Jefferson T, Del Mar CB, Dooley L, Ferroni E, Al-Ansary LA, Bawazeer GA, et al. Physical interventions to interrupt or reduce the spread of respiratory viruses. Cochrane Database Syst Rev 2011; (7):CD006207.

10. Rothe C, Schunk M, Sothmann P, Bretzel G, Froeschl G, Wallrauch C, et al. Transmission of 2019-nCoV infection from an asymptomatic contact in Germany. N Engl J Med 2020; 382:970-1.

11. Yu P, Zhu J, Zhang Z, Han Y, Huang L. A familial cluster of infection associated with the 2019 novel coronavirus indicating possible personto-person transmission during the incubation period. J Infect Dis 2020; [Epub ahead of print].

12. Li C, Ji F, Wang L, Wang L, Hao J, Dai M, et al. Asymptomatic and human-to-human transmission of SARS-CoV-2 in a 2-family cluster, Xuzhou, China. Emerg Infect Dis 2020; [Epub ahead of print].

13. Rache B, Rocha R, Nunes L, Spinola P, Malik AM, Massuda A. Necessidades de infraestrutura do SUS em preparo à COVID-19: leitos de UTI, respiradores e ocupação hospitalar. São Paulo: Instituto de Estudos para Políticas de Saúde; 2020. (Nota Técnica, 3).

14. Wu Z, McGoogan JM. Characteristics of and important lessons from the coronavirus disease 2019 (COVID-19) outbreak in China: summary of a report of 72,314 cases from the Chinese Center for Disease Control and Prevention. JAMA 2020; [Epub ahead of print].

15. Ministério da Saúde. Resolução no 1, de 29 de setembro de 2011. Estabelece diretrizes gerais para a instituição de Regiões de Saúde no âmbito do Sistema Único de Saúde (SUS), nos termos do Decreto no 7.508, de 28 de junho de 2011. Diário Oficial da União 2011; 30 set.

16. Collins LM, Lanza ST. Latent class and latent transition analysis. Danvers: John Wiley \& Sons Inc.; 2010.

17. Cavalcanti AD, Moreira RS, Diniz GTN, Vilela MBR, Silva VL. Active aging and its interface with social determinants of health. Geriatr Gerontol Aging 2018; 12:15-23.

18. Muthén LK, Muthén BO. Mplus user's guide. 8th Edition. Los Angeles: Muthén \& Muthén; 2017.

19. Berlin KS, Williams NA, Parra GR. An introduction to latent variable mixture modeling (part 1): overview and cross-sectional latent class and latent profile analyses. J Pediatr Psychol 2014; 39:174-87.
20. Lubke GH, Muthén B. Investigating population heterogeneity with factor mixture models Psychol Methods 2005; 10:21-39.

21. Nylund KL, Asparouhov T, Muthén BO. Deciding on the number of classes in latent class analysis and growth mixture modeling: a Monte Carlo Simulation Study. Structural Equation Modeling 2007; 14:535-69.

22. Kulldorff MA. Spatial scan statistic. Communications in Statistics - Theory and Methods 1997; 26:1481-96.

23. Bailey TC, Gatrell AC. Interactive spatial data analysis. Harlow: Longman; 1995.

24. Ministério da Saúde. Introdução à estatística espacial para a saúde pública. Brasília: Ministério da Saúde; 2007. (Série B. Textos Básicos de Saúde) (Série Capacitação e Atualização em Geoprocessamento em Saúde, 3).

25. Gao Q, Hu Y, Dai Z. The epidemiological characteristics of 2019 novel coronavirus diseases (COVID-19) in Jingmen, Hubei, China. medRxiv 2020; 10 mar. https://www.medrxiv. org/content/10.1101/2020.03.07.20031393v1.

26. Hick J, Biddinger P. Novel coronavirus and old lessons: preparing the health system for the pandemic. N Engl J Med 2020; [Epub ahead of print].

27. Emanuel EJ, Persad G, Upshur R, Thome B, Parker M, Glickman A, et al. Fair allocation of scarce medical resources in the time of Covid-19. N Engl J Med 2020; [Epub ahead of print].

28. Ventilator Document Workgroup; Ethics Subcommittee of the Advisory Committee to the Director; Centers for Disease Control and Prevention. Ethical considerations for decision making regarding allocation of mechanical ventilators during a severe influenza pandemic or other public health emergency. Atlanta: Centers for Disease Control and Prevention; 2011.

29. Moodley K, Hardie K, Selgelid MJ, Waldman RJ, Strebel P, Rees H, et al. Ethical considerations for vaccination programmes in acute humanitarian emergencies. Bull World Health Organ 2013; 91:290-7.

30. Ran L, Chen X, Wang Y, Wu W, Zhang L, Tan $\mathrm{X}$. Risk factors of healthcare workers with corona virus disease 2019: a retrospective cohort study in a designated hospital of Wuhan in China. Clin Infect Dis 2020; [Epub ahead of print].

31. Wang D, de Lucca-Silveira M. Escolhas dramáticas em contextos trágicos: alocação de vagas em UTI durante a crise da COVID-19. São Paulo: Instituto de Estudos para Políticas de Saúde; 2020. (Nota Técnica, 5). 


\section{Abstract}

In response to the accelerated increase in the number of COVID-19 cases, countries must increase their supply of beds in intensive care units (ICUs). Respiratory diseases, neoplasms, cardiopathies and hypertension, and diabetes are associated with higher COVID-19 case-fatality. The study aimed to identify the regions of Brazil with higher specific mortality rates from these comorbidities and the regions with the greatest shortage of ICU beds and mechanical ventilators. A cross-sectional ecological study was performed in which the units of analysis were the country's Health Regions. Data were obtained from Brazilian Health Informatics Department - DATASUS (National Registry of Healthcare Establishments - 2019, Mortality Information Systems - 2017, and Population Projections - 2017). We calculated the disease group-specific mortality rates for hypertension, neoplasms, diabetes, cardiac diseases, respiratory diseases and the rates of total ICU beds, private ICU beds, ICU beds in the Brazilian Unified $\mathrm{Na}$ tional Health System (SUS), and ventilators in the SUS, per 100,000 inhabitants. The mortality profile was determined by latent profiles analysis, and the cluster analysis of ICU beds and ventilators used the spatial scan method. Kernel maps were constructed for the data's visualization. Level of significance was set at 5\%. Four latent mortality profiles were observed. The Health Regions with the highest mean mortality rates were located in regions with shortages of ICU beds and ventilators, especially in parts of the Northeast, Southeast, and South of Brazil. The spatial localization of regions with both the highest mortality and shortages of ICU beds/ventilators requires attention by policymakers and public planners to deal efficiently and fairly with the COVID-19 epidemic in Brazil.

COVID-19; Intensive Care Units; Mechanical Ventilators; Latent Class Analysis; Mortality

\section{Resumen}

El acelerado aumento en el número de casos de la enfermedad por el nuevo coronavirus (COVID-19) exige que los paises aumenten sus plazas en las unidades de cuidados intensivos (UCI). Enfermedades respiratorias, neoplasias, cardiopatías, hipertensión y diabetes aumentan su letalidad. El estudio tuvo como objetivo identificar tanto las regiones con mayores tasas de mortalidad específica por estas enfermedades, como las que tenían mayor escasez de UCI y ventiladores pulmonares. Se realizó un estudio ecológico transversal, la unidad de análisis fueron las regiones de salud en Brasil. La fuente de datos fue el Departamento de Informática del SUS - DATASUS (Registro Nacional de Establecimientos de Salud - 2019, Sistemas de Información de Mortalidad - 2017 y Proyecciones Poblacionales - 2017). Se calcularon las tasas por 100 mil habitantes de mortalidad especifica para hipertensión, neoplasias, diabetes, enfermedades cardiacas y respiratorias, camas de UCI total, camas de UCI privadas, camas de UCI del Sistema Unico de Salud (SUS) y ventiladores del SUS. El perfil de mortalidad se determinó por el análisis de perfiles latentes y el análisis de clústeres de las camas y ventiladores fue realizado por el método de análisis espacial. Se construyeron mapas de Kernel para la visualización de los datos. El nivel de significancia fue de un 5\%. Se observaron 4 perfiles latentes de mortalidad. Las regiones de salud con las mayores medias en la mortalidad se localizaron en regiones cuya escasez de camas de UCI y ventiladores se visualizó, especialmente en partes de las regiones Nordeste, Sudeste y Sur. La localización espacial de las regiones con mayor mortalidad y con escasez de camas de UCI/ventiladores requiere la atención de los gestores y planificadores públicos para el combate eficiente y ecuánime de la epidemia en Brasil.

COVID-19; Unidades de Cuidados Intensivos; Ventiladores Mecánicos; Análisis de Clases Latentes; Mortalidad
Recebido em 13/Abr/2020

Versão final reapresenatda em 22/Abr/2020 Aprovado em 23/Abr/2020 